\title{
Plastic strain curl theory
}

\author{
S. Xia and T. C. Wang, Beijing, China \\ Received December 12, 2003; revised May 15, 2004 \\ Published online: October 4, 2004 (C) Springer-Verlag 2004
}

\begin{abstract}
Summary. The main mechanisms for the hardening of metal materials are the multiplication, accumulation and interaction of dislocations. The dislocation density tensor can be decomposed into two parts: one is the plastic strain curl tensor and the other is the plastic curvature tensor. The influence of the plastic curvature can be characterized by the interaction between the Cauchy's stresses and the couple stresses. The plastic strain curl is supposed to play the most important role for the stress level. Three rotational degrees of freedom $\omega_{i}$, named as micro rotation, are introduced besides the displacement components $u_{i}$. Micro rotations $\omega_{i}$ have no direct dependence upon $u_{i}$ while the material rotation $\theta=\nabla \times \mathbf{u} / 2$. The generalized normality law is used to describe constitutive relations of Cauchy's stresses versus strains and couple stresses versus curvatures. Plastic strain curl is incorporated into the instantaneous tangent modulus. In this way, the generalized equivalent stress is no longer a single-variable function of the generalized equivalent strain. The plastic strain energy density is no longer determined by the generalized equivalent strain solely, too.

Based on the present theory, an FEM program is developed to simulate the microindentation tests on Copper and Tungsten. The calculated hardness is observed to elevate as the indent depth decreases. The calculated results agree well with the experimental data. The crack tip field for small scale yielding condition is also studied. The calculated results clearly show that the stress level near the crack tip with plastic strain curl effects is considerably higher than that in the conventional plasticity theory. The singularity of the mean stress near the crack tip is nearly equal to the square-root singularity, and the singularity of the effective stress field is slightly greater than the square-root singularity. Consequently, the singularity of stress components is also slightly greater than the square-root singularity. The $J$-integral is observed to be essentially path independent.
\end{abstract}

\section{Introduction}

The work hardening of metallic materials is caused by the storage and interaction of dislocations. According to Taylor's hardening law, the flow strength of the material is related to the dislocation density in the material by the following formula:

$\sigma=C G B \sqrt{\rho}$,

where $C$ is a constant coefficient, $G$ is the shear modulus and $b$ is the magnitude of Burger's vector $\mathbf{b}$. The dislocations are stored as two types: the statistically stored dislocations and the geometrically necessary dislocations [1], [2]. Then, the flow strength is

$\sigma=C G B \sqrt{\rho_{s}+\rho_{G}}=C G B \sqrt{\rho_{s}} \sqrt{1+\frac{\rho_{G}}{\rho_{s}}}$,

where $\rho_{S}$ is the statistically stored dislocation density and $\rho_{G}$ is the geometrically necessary dislocation density. 
If the geometrically necessary dislocation density is negligibly small, the flow strength can be expressed as

$\sigma=C G B \sqrt{\rho_{s}}$.

On the other hand, the uniaxial stress-strain law of the classical theory of plasticity can be written as

$\sigma=\sigma_{0} A(\varepsilon)$

or

$\sigma=\sigma_{0} A\left(\varepsilon_{e p}\right)$,

where $\sigma_{0}$ is the yield stress, $A$ is the work hardening function, $\sigma_{e}$ is the equivalent stress and $\varepsilon_{e p}$ is the equivalent plastic strain. From Eqs. (1.3), (1.4.1) and (1.4.2), one can see that the statistically stored dislocation density is a function of the equivalent plastic strain.

In the conventional theories of plasticity, only the influence of statistically stored dislocations is considered. Comparing with the statistically stored dislocation density, the geometrically necessary dislocation density is negligible at macro scales. That is why the conventional theories of plasticity are successfully used at those scales.

The geometrically necessary dislocation density is related to the inhomogeneous plastic strain [1]. Once the size of the plastic deformation zone is of a few microns or less, the plastic strains vary sharply in a small zone, and then the geometrically necessary dislocation density cannot be neglected anymore. Therefore, the local stress level may be much higher than the calculated results using a conventional plasticity model.

The phenomenon of increase of material strength at micron scale was reported in numerous microindentation studies [6]. Pyramidal indents whose depths exceed fifty microns generally produce size-independent hardness values in most metals, just consistent with the conclusion of the conventional theory of plasticity. Nevertheless, the mentioned microindentation tests showed an increase in hardness with decreasing depth of penetration for depths less than ten microns. This phenomenon is known as the indentation size effect. Since the conventional theory of plasticity involved no material length scales, it cannot be used to explain the size effect.

Another instance of stress elevation was revealed in the stress field near a crack tip. In a remarkable experimental work, Elssner et al. [7] measured both the macroscopic fracture toughness and the atomic separating work of an interface between a single crystal of Niobium and a Sapphire single crystal. According to their experimental results, Hutchinson [8] concluded that the stress level needed to produce atomic decohesion of a lattice or a strong interface were generally considerably larger than 5 times the tensile yield stress $\sigma_{0}$ of the metal. However, the maximum stress level that could be achieved ahead of the crack tip was not greater than about $4 \sigma_{0}-5 \sigma_{0}$ according to models based on the conventional theory of plasticity. It is evident that the conventional plasticity theory is inadequate at the small scales involved in the crack tip deformation.

In order to explain the experimental results, it is necessary to develop a continuum plastic theory with intrinsic material length scales.

Fleck, Hutchinson and their co-workers proposed a version of couple stress theory (CS theory) [9], [10]. Shu and Fleck [11] investigated the microindentation problem with the CS theory but they failed to simulate the test. The asymptotic analysis [12] and FEM calculation [14], [16] also found that the stress level near a crack tip estimated by the CS theory was essentially the same as that by conventional plasticity. The reason of these phenomena might lie in the fact that the stretch gradient was not considered in the CS theory. 
Then a general strain gradient theory of plasticity (SG theory) was developed by Fleck and Hutchinson [17] to introduce the effect of stretch gradient. Begley and Hutchinson [18] simulated successfully the microindentation test with the SG theory and determined the length scales in that theory by fitting the experimental data. Wei and Hutchinson [19], Chen et al. [20] and Shi et al. [21] used the SG theory to study the crack tip field. The elevation of stress level was indeed observed in their analysis. Nevertheless, as a mode-I crack tip was approached, the traction was found to switch to compressive [20]. It was therefore concluded by Chen et al. [20] that the asymptotic crack tip field in the SG theory had no domain of physical validity.

Gao, Huang and their co-workers [22], [24] presented a mechanism-based strain gradient theory (MSG theory). Based on the MSG plasticity theory, Huang et al. [25] investigated the microindentation problem. Shi et al. [26] investigated the asymptotic crack tip field of MSG theory and concluded that its near crack tip field was inseparable. Jiang et al. [27] presented the crack tip field with finite element analysis of MSG theory. They observed the stress elevation and found that the stress singularity exceeded or equaled the square-root singularity.

Third-order stresses were introduced in both the SG theory and MSG theory. As a result, the governing equations become quite complicated and the extra complex boundary condition must be introduced. Retaining the essential structure of conventional plasticity, Acharya and Bassani [28] concluded that a possible formulation is a flow theory with strain gradient measures incorporated into the instantaneous hardening functions. However, they did not give a systematic way of constructing the tangent modulus to validate this framework. Following their thought, Chen and Wang [29] established a new hardening law based on the incremental version of conventional $J_{2}$ deformation theory. The effective strain gradient was only a parameter to influence the tangent modulus in that hardening law. Chen and Wang [30], [31] established a new version of the phenomenological strain gradient theory for crystalline solids. Stretch gradients were introduced in a similar way as that by Chen and Wang [29]. Based on the new theory, both the asymptotic fields [32], [33] and the full field solution [34] were studied. Xia et al. [35] developed a simplified theory of strain gradient. The essential structure of conventional $J_{2}$ deformation theory was maintained in the simplified theory. Neither couple stress nor higher-ordered stress was introduced. The strain gradient measure influenced the instantaneous tangent modulus only. Plane-strain mode-I crack tip field was investigated with the simplified theory. The stress elevation was observed.

A plastic strain curl theory is presented in this paper. The initial idea of the new theory comes from the continuum theory of dislocation [36]. In that theory, the dislocation density tensor can be written as

$\boldsymbol{\rho}=-\operatorname{rot} \boldsymbol{\varepsilon}^{p}-\left(\operatorname{tr} \tilde{\boldsymbol{\chi}}^{p}\right) \mathbf{I}+\left(\tilde{\boldsymbol{\chi}}^{p}\right)^{T}$,

where $\varepsilon^{p}$ is the plastic strain tensor, $\tilde{\chi}^{p}$ is the plastic curvature tensor, $\mathbf{I}$ is the unit tensor. Equation (1.5.1) is deduced from the displacement incompatibility introduced by dislocations. In fact, at the micro scale, the statistically stored dislocations rapidly accumulate by trapping one another in a random distribution way and introduce no incompatibility into the solids at macro scales. It means that the densities of statistically stored dislocations only correspond to the uniform deformation at macro scales. Only the geometrically necessary dislocations are required for the nonuniform deformation at macro scales, which is consistent with Eq. (1.5.1). Hence, the dislocation density calculated in conformity to Eq. (1.5.1) can only be the density of geometrically necessary dislocations, i.e., 
$\boldsymbol{\rho}_{G}=-\operatorname{rot} \boldsymbol{\varepsilon}^{p}-\left(\operatorname{tr} \tilde{\boldsymbol{\chi}}^{p}\right) \mathbf{I}+\left(\tilde{\boldsymbol{\chi}}^{p}\right)^{T}$.

It is evident that the density tensor of geometrically necessary dislocations can be decomposed into two parts: one is the plastic strain curl tensor and another is related to the plastic curvature tensor. One can imagine that the work conjugate of the curvature tensor is the couple stress. Hence, the effect of the plastic curvature tensor on the plastic deformation can be described by introducing the couple stress. Thus, the plastic strain curl will play the key role in the new theory.

Plastic strain curl theory is constructed based on general couple stress theory [37]-[42]. Micro rotations $\omega_{i}$ are introduced. Curvatures $\chi_{i j}$ are the gradients of micro rotations $\omega_{i}$. The constitutive relations of Cauchy's stresses versus strains and couple stresses versus curvatures are deduced from the generalized normality law.

Section 2 gives a brief summary on the governing equations and boundary conditions of plastic strain curl theory. The finite element formulation is introduced in Sect.3, and the calculated results are discussed in Sect. 4.

In the remainder of the present paper, if not claimed, a repeated suffix denotes summation over 1 to 3 . A comma in the suffix indicates a partial derivative with respect to a Cartesian coordinate.

\section{Plastic strain curl theory}

All materials consist of billion discrete micro particles (e.g., atoms, molecules, ions, etc.). The location changes of all the particle centers form a displacement field, denoted by $\mathbf{u}$. Material rotation $\theta$ is dependent on the displacement, i.e., $\theta=\nabla \times \mathbf{u} / 2$. The micro rotation $\omega$ has no direct dependence upon the displacement $\mathbf{u}$. It is the angular displacement of a particle. In fact, micro rotation $\omega$ is the sum of material rotation $\theta$ and relative rotation $\phi$. The relation between micro rotation $\omega$ and material rotation $\theta$ is illustrated in Fig. 1. In fact, suppose that the material particle centers after deformation form a deformed lattice and the material rotation $\theta$ will reflect the rotation of the deformed lattice which is completely determined by the displacements of the material particle centers while the micro rotation $\omega$ will be the rotation of the material particle itself.
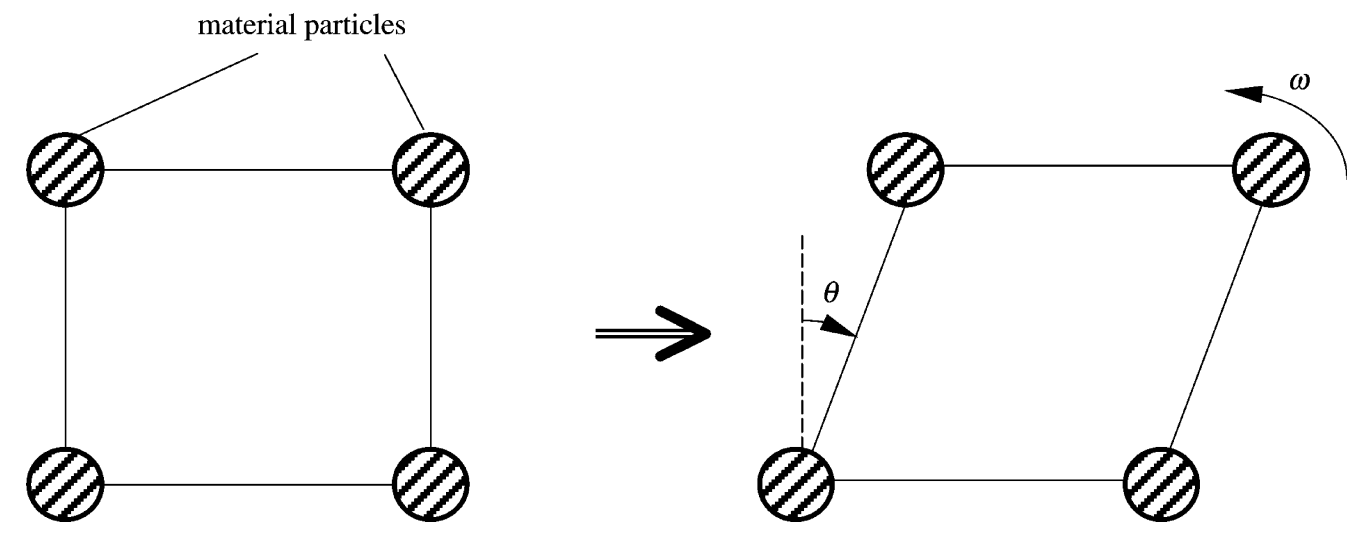

Fig. 1. Relation between micro rotation $\omega$ and material rotation $\theta$ 
In the continuum theory of dislocations [36], the curvature tensor $\tilde{\chi}$ is defined as the gradient of material rotation $\theta$, i.e.,

$\tilde{\chi}_{i j}=\theta_{i, j}$.

The plastic curvature tensor $\tilde{\chi}_{i j}^{p}$ in continuum theory of dislocations is defined in the form

$d \theta_{i}^{p}=\tilde{\chi}_{i j}^{p} d x_{j}$,

where $\theta_{i}^{p}$ is the plastic material rotation. In the plastic strain curl theory, however, the curvature tensor $\chi$ is defined as the gradient of micro rotation $\omega$, i.e.,

$\chi_{i j}=\omega_{i, j}$.

This definition is different from the definition in the continuum theory of dislocations. Let us consider the plastic curvature tensor. As shown in Fig. 2, during the plastic deformation, the two groups of micro particles will move mutually on the opposite sides of a slip plane without relative rotations. Therefore the plastic relative rotation $\phi^{p}=0$. Thus it is evident that

$\omega_{i}^{p}=\theta_{i}^{p}, \quad d \omega_{i}^{p}=\chi_{i j}^{p} d x_{j}, \quad \chi_{i j}^{p}=\tilde{\chi}_{i j}^{p}$,

where $\omega_{i}^{p}$ is the plastic micro rotation, and $\chi_{i j}^{p}$ is the plastic curvature tensor in the plastic strain curl theory.

In the present theory, only the symmetric part of Cauchy's stress $\sigma$ and couple stress $\mathbf{m}$ are considered. The antisymmetric part of Cauchy's stress is supposed to be negligibly small. If body forces and body couples are absent, the equilibrium equation within body $V$ gives

$$
\begin{aligned}
\sigma_{i j, j} & =0, \\
l_{C S}^{-1} m_{i j, j} & =0,
\end{aligned}
$$

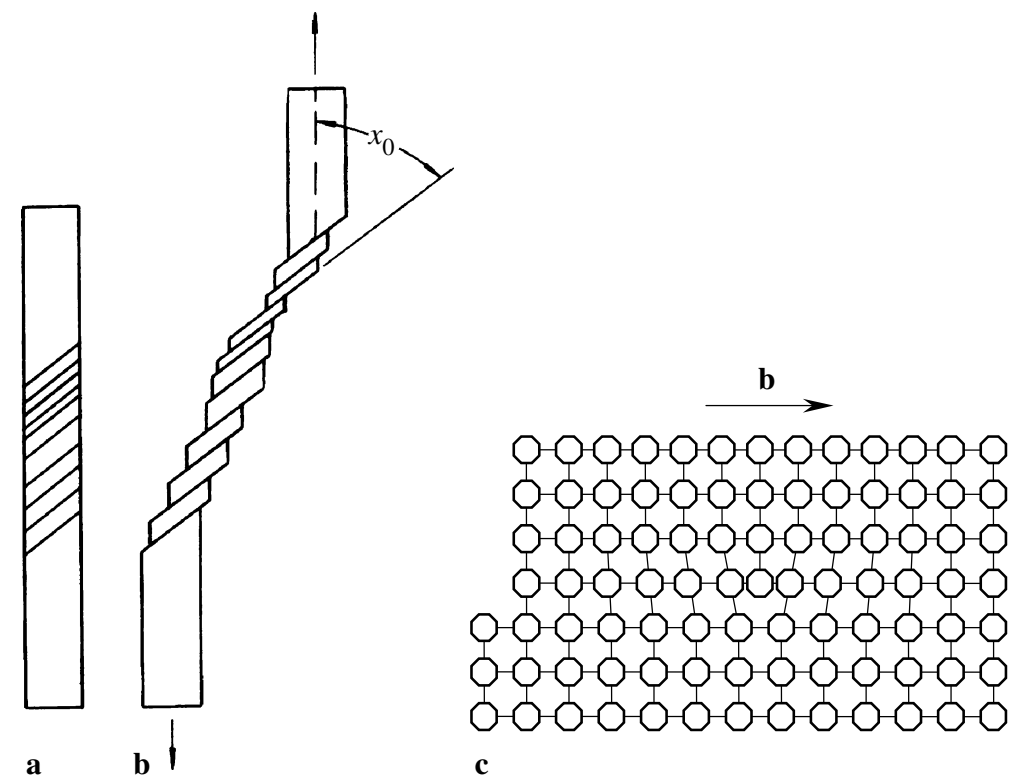

Fig. 2. Slip of crystalline materials; a Crystal before the slips. The inclined lines stand for the possible slip surfaces; b Crystal after the slips. The portions divided by slip surfaces move mutually; c Sketch of a dislocation. The two groups of atoms move mutually on the opposite sides without relative rotation. Note that (b) denotes the Burger's vector 
where $\sigma_{i j}$ is the stress, $m_{i j}$ is the couple stress and $l_{C S}$ is an intrinsic material length scale which characterizes the size of the three-dimensional dislocation network. The three-dimensional dislocation network is supposed to be formed during the manufacturing process, which is related to the smallest nonuniform plastic micro rotation zone.

Traction equilibrium on the surface $S$ of the body $V$ implies:

$\sigma_{i j} n_{j}=t_{i}$,

$m_{i j} n_{j}=q_{i}$

where $n_{i}$ is the outer normal of the surface $S$, and $t_{i}$ and $q_{i}$ are surface tractions and surface torque per unit area, respectively.

The constitutive equations of plastic strain curl theory can be obtained through an analogy with the conventional theory of plasticity. According to the normality law in conventional $J_{2}$ deformation theory, plastic strains can be written as

$\varepsilon_{i j}^{p}=\frac{3 \varepsilon_{e p}}{2 \sigma_{e}} s_{i j}$,

where $s_{i j}$ is the deviatoric stress, $\sigma_{e}$ is the equivalent stress, and $\varepsilon_{e p}$ is the equivalent plastic strain. Equation (2.7) can be easily rewritten as

$s_{i j}=\frac{2 \sigma_{e}}{3 \varepsilon_{e p}} \varepsilon_{i j}^{p}$.

In another hand, the relation between mean stress and volume strain is

$\frac{\sigma_{k k}}{3}=K \varepsilon_{k k}$,

where $\varepsilon_{i j}$ is strain and $K$ is volume modulus. Combining Eqs. (2.8) and (2.9), the constitutive equations in the conventional $J_{2}$ deformation theory are written as

$\sigma_{i j}=\frac{2 \sigma_{e}}{3 \varepsilon_{e p}} \varepsilon_{i j}^{p}+\delta_{i j} K \varepsilon_{k k} \quad\left(\sigma_{e} \geq \sigma_{0}\right)$,

where $\delta_{i j}$ is Kronecker's delta. In a similar way, the constitutive equations in plastic curl theory can be obtained, i.e.,

$$
\begin{aligned}
\sigma_{i j} & =\frac{2 \sum_{e}}{3 E_{e p}} \varepsilon_{i j}^{p}+\delta_{i j} K \varepsilon_{k k} \\
l_{C S}^{-1} m_{i j} & =\frac{2 \sum_{e}}{3 E_{e p}} l_{C S} \chi_{i j}^{p}+\delta_{i j} K_{1} l_{C S} \chi_{k k}
\end{aligned} \quad\left(\Sigma_{e}>\sigma_{0}\right),
$$

where $K_{1}$ is called the bend-torsion volume modulus, which is defined in the way that

$l_{C S}^{-1} m_{k k}=3 K_{1} l_{C S} \chi_{k k}$.

A generalized normality law has been employed in Eq. (2.11.1). Generalized equivalent stress $\Sigma_{e}$ and generalized equivalent plastic strain $E_{e p}$ have taken the places of the equivalent stress $\sigma_{e}$ and equivalent plastic strain $\varepsilon_{e p}$ in Eq. (2.10), respectively. The expressions of generalized stress $\Sigma_{e}$ and generalized plastic strain $E_{e p}$ are

$\Sigma_{e}^{2}=\sigma_{e}^{2}+l_{C S}^{-2} m_{e}^{2}, \quad E_{e p}^{2}=\varepsilon_{e p}^{2}+l_{C S}^{2} \chi_{e p}^{2}$, 
where $m_{e}=\left(3 m_{i j}^{\prime} m_{i j}^{\prime} / 2\right)^{1 / 2}$ is the equivalent couple stress, $\chi_{e p}=\left(2 \chi_{i j}^{p} \chi_{i j}^{p} / 3\right)^{1 / 2}$ is the equivalent plastic curvature and $m_{i j}^{\prime}$ is the deviatoric couple stress.

The incremental version of Eq. (2.11.1) is

$$
\begin{aligned}
\dot{\sigma}_{i j} & =\frac{2 \sum_{e}}{3 E_{e p}} \dot{\epsilon}_{i j}^{p}+\frac{2 \dot{\sum}_{e}}{3 E_{e p}} \varepsilon_{i j}^{p}-\frac{2 \sum_{e}}{3 E_{e p}^{2}} \dot{E}_{e p} \varepsilon_{i j}^{p}+\delta_{i j} K \dot{\varepsilon}_{k k} \\
l_{C S}^{-1} \dot{m}_{i j} & =\frac{2 \sum_{e}}{3 E_{e p}} l_{C S} \dot{\chi}_{i j}^{p}+\frac{2 \dot{\sum}_{e}}{3 E_{e p}} l_{C S} \dot{\chi}_{i j}^{p}-\frac{2 \sum_{e}}{3 E_{e p}^{2}} \dot{E}_{e p} l_{C S} \dot{\chi}_{i j}^{p}+\delta_{i j} K_{1} l_{C S} \dot{\chi}_{k k}
\end{aligned} \quad
$$

Equations (2.11.1) and (2.13) are similar to the constitutive equations of general couple stress theory. However, the plastic curl theory is distinguished from the general couple stress theory by its formulation of instantaneous tangent modulus.

The hardening law in the theory of conventional plasticity is

$\sigma_{e}=\sigma_{0} A\left(\varepsilon_{e p}\right) \quad$ or $\quad \dot{\sigma}_{e}=\sigma_{0} A^{\prime}\left(\varepsilon_{e p}\right) \dot{\varepsilon}_{e p}$,

where $\sigma_{0} A^{\prime}\left(\varepsilon_{e p}\right)$ is the instantaneous tangent modulus in the theory of conventional plasticity. Similarly, the hardening law in general couple stress theory is

$\Sigma_{e}=\sigma_{0} A^{\prime}\left(E_{e p}\right) \quad$ or $\quad \dot{\Sigma}_{e}=\sigma_{0} A^{\prime}\left(E_{e p}\right) \dot{E}_{e p}$,

where $\sigma_{0} A^{\prime}\left(E_{e p}\right)$ is the instantaneous tangent modulus in general couple stress theory.

The incremental version of the hardening law in plastic strain curl theory is different from Eq. (2.15), i.e.,

$\dot{\sum}_{e}=\sigma_{0} H\left(E_{e p}, l_{1} \eta_{e p}\right) \dot{E}_{e p}=\sigma_{0} A^{\prime}\left(E_{e p}\right) R\left(E_{e p}, l_{1} \eta_{e p}\right) \dot{E}_{e p}$,

where $\sigma_{0} H\left(E_{e p}, l_{1} \eta_{e p}\right)$ is the instantaneous tangent modulus in plastic strain curl theory where $l_{1}$ is the second intrinsic length scale which characterizes the size of the micro dislocation structure. The micro dislocation structure is supposed to be formed during the manufacturing process which is related to the smallest nonuniform plastic deformation zone. Since the plastic strain curl takes a negligible effect at the large scale, the value of $R\left(E_{e p}, l_{1} \eta_{e p}\right)$ should be unity when the characterized scale of the realistic nonuniform plastic deformation zone is far greater than $l_{1}$. Hinted by Eq. (1.2) and following the proposal of Chen and Wang [29], [34], and Xia et al. [35], the expression of the coefficient $R\left(E_{e p}, l_{1} \eta_{e p}\right)$ is supposed to be

$R\left(E_{e p}, l_{1} \eta_{e p}\right)=\sqrt{1+\frac{l_{1} \eta_{e p}}{E_{e p}}}$,

where $\eta_{e p}$ is a measure of both the plastic strain curl and the plastic curvature tensor, i.e.,

$\eta_{e p}=\eta_{e p}\left(\xi_{i j}^{p} \xi_{i j}^{p}, \xi_{i j}^{p} \xi_{j i}^{p}, \chi_{i j}^{p} \chi_{i j}^{p}, \chi_{i j}^{p} \chi_{j i}^{p}, \chi_{k k}^{p}\right)$,

where $\xi^{p}=\operatorname{rot} \varepsilon^{p}$ is the plastic strain curl tensor, and its component expression is

$\xi_{i j}^{p}=e_{i k l} \varepsilon_{j l, k}^{p}$,

where $e_{i j k}$ is the permutation tensor. Since the effect of the plastic curvature tensor $\chi^{p}$ can be described by introducing the couple stress, $\eta_{e p}$ can be supposed to be a scalar function of plastic strain curl only, i.e.,

$\eta_{e p}=\sqrt{\xi_{i j}^{p} \xi_{i j}^{p}+c \xi_{i j}^{p} \xi_{j i}^{p}}$

where $c$ is a dimensionless constant. 
It is clear that the coefficient $R\left(E_{e p}, l_{1} \eta_{e p}\right)$ manifests the influence of plastic strain curl (or, in other words, the dislocation density) on the instantaneous tangent modulus. This is one of the key characters of plastic strain curl theory. As another important character, because of the existence of the coefficient $R\left(E_{e p}, l_{1} \eta_{e p}\right)$, the generalized equivalent stress $\Sigma_{e}$ is no longer a single-variable function of the generalized equivalent plastic strain $E_{e p}$, and the strain energy density $w$ is no longer determined by the strain $\varepsilon$ and curvature tensor $\chi$ only. Both the generalized equivalent stress $\Sigma_{e}$ and the strain energy density $w$ depend on the loading history.

An exponent law of hardening is employed in the present work. The expression of the work hardening function $A\left(\varepsilon_{e p}\right)$ is

$A\left(\varepsilon_{e p}\right)=\left(1+\frac{\varepsilon_{e p}}{\varepsilon_{0}}\right)^{n}$,

where $n$ is the hardening exponent, $\varepsilon_{0}$ is a reference strain, and it is taken as the equivalent strain at which the material yields, i.e.,

$\varepsilon_{0}=\frac{\sigma_{0}}{3 \mu}$,

where $\mu$ is the shear modulus.

After a laborious deduction, Eq. (2.13) can be rewritten in another form. Then the complete constitutive equations of plastic strain curl theory, with both the elastic and plastic ranges, can be written as

$$
\begin{aligned}
& \left\{\begin{array}{l}
\dot{\sigma}_{i j}=\left[2 \mu \delta_{i k} \delta_{j l}+\left(K-\frac{2 \mu}{3}\right) \delta_{i j} \delta_{k l}\right] \dot{\varepsilon}_{k l} \\
l_{C S}^{-1} \dot{m}_{i j}=\left[2 \mu \delta_{i k} \delta_{j l}+\left(K_{1}-\frac{2 \mu}{3}\right) \delta_{i j} \delta_{k l}\right] l_{C S} \dot{\chi}_{k l}
\end{array} \quad\left(\Sigma_{e}<\sigma_{0}\right),\right. \\
& \left\{\begin{array}{l}
\dot{\sigma}_{i j}=D_{i j k l}^{(1)} \dot{\varepsilon}_{k l}+D_{i j k l}^{(2)} l_{C S} \dot{\chi}_{k l} \\
l_{C S}^{-1} \dot{m}_{i j}=D_{k l i j}^{(2)} \dot{\varepsilon}_{k l}+D_{i j k l}^{(3)} l_{C S} \dot{\chi}_{k l}
\end{array} \quad\left(\Sigma_{e} \geq \sigma_{0}\right),\right.
\end{aligned}
$$

where

$$
\begin{aligned}
& D_{i j k l}^{(1)}=C_{1} \delta_{i k} \delta_{j l}+\left(K-\frac{C_{1}}{3}\right) \delta_{i j} \delta_{k l}+C_{2} \frac{\varepsilon_{i j}^{p} \varepsilon_{k l}^{p}}{E_{e p}^{2}}, \\
& D_{i j k l}^{(2)}=C_{2} \frac{l_{C S} \varepsilon_{i j}^{p} \chi_{k l}^{p}}{E_{e p}^{2}} \\
& D_{i j k l}^{(3)}=C_{1} \delta_{i k} \delta_{j l}+\left(K_{1}-\frac{C_{1}}{3}\right) \delta_{i j} \delta_{k l}+C_{2} \frac{l_{C S}^{2} \chi_{i j}^{p} \chi_{k l}^{p}}{E_{e p}^{2}}, \\
& C_{1}=\frac{2 \mu B_{1}}{2 \mu+B_{1}} \\
& C_{2}=\frac{4 \mu^{2} B_{2} E_{e p}^{2}}{\left(2 \mu+B_{1}\right)\left(2 \mu+B^{*}\right)} \\
& B^{*}=B_{1}+B_{2}\left(I_{1}+I_{2}\right), \\
& B_{1}=\frac{2 \Sigma_{e}}{3 E_{e p}}
\end{aligned}
$$


$B_{2}=\frac{4}{9 E_{e p}^{2}}\left(\sigma_{0} H\left(E_{e p}, l_{1} \eta_{e p}\right)-\frac{\Sigma_{e}}{E_{e p}}\right)$

$I_{1}=\varepsilon_{i j}^{p} \varepsilon_{i j}^{p}, \quad I_{2}=l_{C S}^{2} \chi_{i j}^{p} \chi_{i j}^{p}$.

It should be emphasized that the stiffness coefficients defined in Eq. (2.22) will not approach infinity when the generalized equivalent plastic strain $E_{e p}$ approaches zero. In fact, substitute Eq. (2.25.1) into Eq. (2.23.1), and the expression of the coefficient $C_{1}$ can be changed to

$C_{1}=\frac{2 \mu \frac{2 \Sigma_{e}}{3 E_{e p}}}{2 \mu+\frac{2 \Sigma_{e}}{3 E_{e p}}}=\frac{2 \mu \Sigma_{e}}{3 \mu E_{e p}+\Sigma_{e}}$.

It is evident that the value of $C_{1}$ will be $2 \mu$ when the generalized equivalent plastic strain $E_{e p}$ equals zero. Then substituting Eqs. (2.24-2.26) into Eq. (2.23.2), one will get

$C_{2}=\frac{4 \mu^{2} \frac{4}{9 E_{e p}^{2}}\left(\sigma_{0} A^{\prime}\left(E_{e p}\right) R\left(E_{e p}, l_{1} \eta_{e p}\right)-\frac{\Sigma_{e}}{E_{e p}}\right) E_{e p}^{2}}{\left(2 \mu+\frac{2 \Sigma_{e}}{3 E_{e p}}\right)\left[2 \mu+\frac{2 \Sigma_{e}}{3 E_{e p}}+\frac{4}{9 E_{e p}^{2}}\left(\sigma_{0} A^{\prime}\left(E_{e p}\right) R\left(E_{e p}, l_{1} \eta_{e p}\right)-\frac{\Sigma_{e}}{E_{e p}}\right)\left(\varepsilon_{i j}^{p} \varepsilon_{i j}^{p}+l_{C S}^{2} \chi_{i j}^{p} \chi_{i j}^{p}\right)\right]}$.

Substituting Eq. (2.12) into Eq. (2.28), the expression of the coefficient $C_{2}$ can be written as

$C_{2}=\frac{4 \mu^{2}\left[\sigma_{0} A^{\prime}\left(E_{e p}\right) E_{e p}-\frac{\Sigma_{e}}{R\left(E_{e p}, l_{1} \eta_{e p}\right.}\right]}{\left(3 \mu E_{e p}+\Sigma_{e}\right)\left[\frac{3 \mu}{R\left(E_{e p}, l_{1} \eta_{e p}\right)}+\sigma_{0} A^{\prime}\left(E_{e p}\right)\right]}$.

When $E_{e p}$ approaches zero, it can be seen from Eqs. (2.19) and (2.17) that if $l_{1} \eta_{e p} \neq 0, A^{\prime}\left(E_{e p}\right)$ will approach $n / \varepsilon_{0}$, and $1 / R\left(E_{e p}, l_{1} \eta_{e p}\right)$ will approach zero, hence the value of $C_{2}$ will approach zero. Furthermore, based on the generalized normality law, it can be concluded that

$\frac{\varepsilon_{i j}^{p}}{E_{e p}}=\frac{3 s_{i j}}{2 \Sigma_{e}}, \quad \frac{l_{C S} \chi_{i j}^{p}}{E_{e p}}=\frac{3 l_{C S}^{-1} m_{i j}}{2 \Sigma_{e}}$.

Therefore,

$\frac{\varepsilon_{i j}^{p} \varepsilon_{k l}^{p}}{E_{e p}^{2}}=\frac{9 s_{i j} s_{k l}}{4 \Sigma_{e}^{2}}, \quad \frac{l_{C S} \varepsilon_{i j}^{p} \chi_{k l}^{p}}{E_{e p}^{2}}=\frac{9 l_{C S}^{-1} s_{i j} m_{k l}^{\prime}}{4 \Sigma_{e}}, \quad \frac{l_{C S}^{2} \chi_{i j}^{p} \chi_{k l}^{p}}{E_{e p}^{2}}=\frac{9 l_{C S}^{-2} m_{i j}^{\prime} m_{k l}^{\prime}}{4 \Sigma_{e}^{2}}$.

They will not be infinite when the generalized equivalent plastic strain $E_{e p}$ approaches zero. Substituting Eq. (2.31) into Eq. (2.22), one obtains

$D_{i j k l}^{(1)}=C_{1} \delta_{i k} \delta_{j l}+\left(K-\frac{C_{1}}{3}\right) \delta_{i j} \delta_{k l}+C_{2} \frac{9 s_{i j} s_{k l}}{4 \Sigma_{e}^{2}}$,

$D_{i j k l}^{(2)}=C_{2} \frac{9 l_{C S}^{-1} s_{i j} m_{k l}}{4 \Sigma_{e}^{2}}$,

$D_{i j k l}^{(3)}=C_{1} \delta_{i k} \delta_{j l}+\left(K_{1}-\frac{C_{1}}{3}\right) \delta_{i j} \delta_{k l}+C_{2} \frac{9 l_{C S}^{-2} m_{i j}^{\prime} m_{k l}^{\prime}}{4 \Sigma_{e}^{2}}$. 


\section{Finite element simulation of microindentation tests}

For a solid in plastic strain curl theory, the principle of virtual work is

$\int_{V}\left(\sigma_{i j} \delta \varepsilon_{i j}+m_{i j} \delta \chi_{i j}\right) \mathrm{d} V-\int_{s}\left(t_{i} \delta u_{i}+q_{i} \delta \omega_{i}\right) \mathrm{d} s=0$.

A stiff conical indenter is employed in the calculation so that the simulation of microindentation can be simplified as an axisymmetric problem, as shown in Fig. 3. Then, the nonvanishing displacement and micro rotation components are

$u_{r}=u_{r}(r, z), \quad u_{z}=u_{z}(r, z), \quad \omega_{\theta}=\omega_{\theta}(r, z)$.

The indenter is supposed to be frictionless, and the nodes in the contact region are constrained to fall on the indenter, with freedom to slide up and down the face of the indenter. Therefore, for small strain theory and shallow indenter considered here, only $u_{z}$ is prescribed and no restriction is assigned to $u_{r}$ [18]. Hence, the following boundary conditions in the contact region are imposed:

$u_{z}(r)=\delta-\frac{r}{\tan \beta}$ and no restriction on $u_{r}$,

where $\delta$ is the penetration depth of the indenter and $\beta$ is the half-angle of the indenter.

A nine-noded isoparametric element is chosen and each node has three degrees of freedom. The specimen in Fig. 3 is divided into 600 elements with 2601 nodes in total.

During the calculation, the depth of the indenter tip $\delta$ increases incrementally. A certain value of the $z$-displacement $u_{z}(r)$ is prescribed to every node in the contact region at each loading increment. Then the $z$-displacements $u_{z}(r)$ of the nodes on the free surface are calculated at the present loading increment in accordance with the coordinate system shown in Fig. 3. If the obtained $z$-displacement $u_{z}(r)$ of a node on the free surface is just greater than the value described in Eq. (3.3), it means that the node has not contacted with the indenter and the calculation will enter the next increment. If the obtained $z$-displacement $u_{z}(r)$ is equal to that value, it means that the node contact, just the right with the indenter and the $z$-displacement of that node will be prescribed in the next increment. If the obtained $z$-displacement $u_{z}(r)$ is smaller, it means that the node is pushed into the interior of the stiff indenter, which is physically unreasonable. Thus all the displacements, stresses, couple stresses, strains and curvatures will be restored to the previous increment, and the calculation will be restarted at a reduced loading increment.

First two samples of Copper investigated by McElhaney et al. [6] are chosen. One sample was strain-hardened and another had been annealed. The strain-hardened sample was polycrystalline with a grain size that was large compared to the size of the large indentations. The

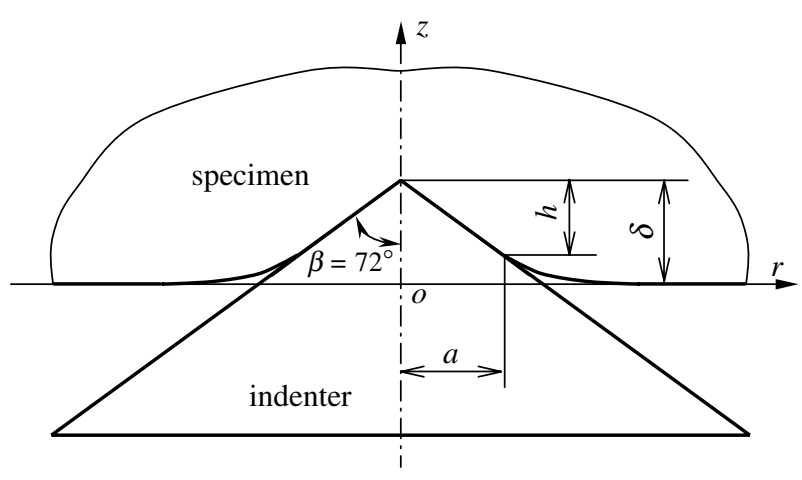

Fig. 3. Calculation model for microindentation test 


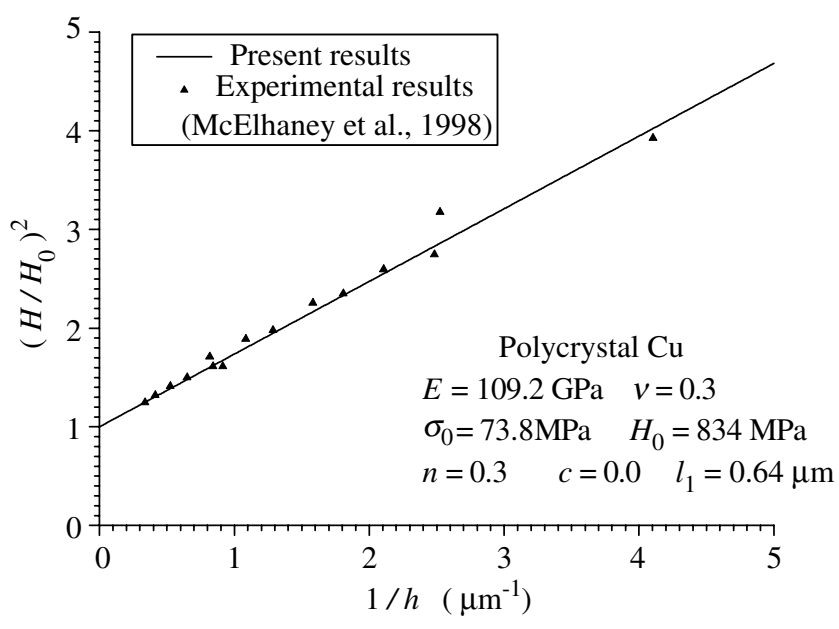

Fig. 4. Simulation of the microindentation test on polycrystalline Copper

annealed sample was a single crystal Copper with the (111) orientation. The material parameters are Young's modulus $E=109.2 \mathrm{GPa}$, Poisson's ratio $v=0.3$ [43], work hardening exponent $n=0.3$ [10], and dimensionless constant $c=0$. Yield stress $\sigma_{0}=73.8 \mathrm{MPa}$ and macroscopic hardness $H_{0}=834 \mathrm{MPa}$ for polycrystal copper, while $\sigma_{0}=38.5 \mathrm{MPa}$ and $H_{0}=581 \mathrm{MPa}$ for single crystal Copper [6]. The indentation simulation results are shown in Figs. 4 and 5, respectively. The FEM results based on the plastic strain curl theory simulate the experimental data successfully. A linear law is shown between the squared hardness $H^{2}$ and the inverse of contacting depth $1 / h$. When the contacting depth $h$ is large enough, the value of hardness $H$ approaches the macroscopic hardness $H_{0}$. The hardness increases as the indent size decreases. The slope of the polycrystalline Copper curve is about 7.36 while the other is about 6.86 , i.e. the former is only slightly higher than the latter. However, the fitting value of material length $l_{1}$ is $0.64 \mu \mathrm{m}$ for polycrystalline copper while $2.42 \mu \mathrm{m}$ for single crystal copper. The obvious difference of the fitting values of material length $l_{1}$ may be caused by the different microstructures of the two types of material. The interfaces divided the polycrystalline body into a large amount of grains. The plastic deformations in the grains introduce a great deal of geometrically necessary dislocations accumulated near the interfaces. This means that the geometrically necessary dislocations are easier to be stored in the polycrystalline materials than in the single crystal materials. Therefore, a relatively smaller material length $l_{1}$ is needed for the

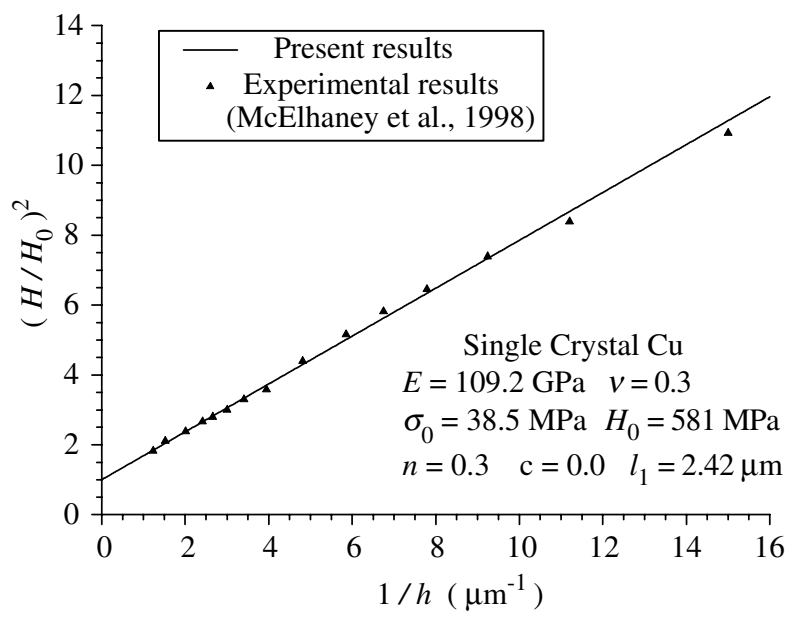

Fig. 5. Simulation of the microindentation test on single crystal Copper 


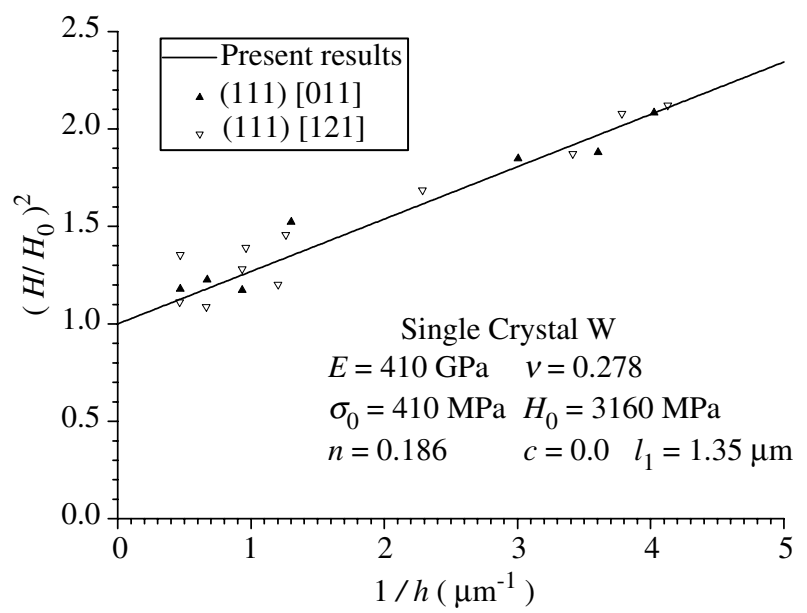

Fig. 6. Simulation of the microindentation test on single crystal Tungsten. (111) plane is indented

polycrystalline materials to get the same effect of stress elevation as the single crystal materials. On the other hand, the characteristic scale of a polycrystalline material is its grain size, i.e., about $0.1-1 \mu \mathrm{m}$. When the size of the nonuniform plastic deformation zone is of the order or smaller than the grain size, the material shows strong size effect. When the size of the nonuniform plastic deformation zone is much larger than the grain size, the size effect is not so evident. For the single crystal materials, however, such a characteristic size should be larger, although its physical background is still not so clear. It should be emphasized that when the number of elements are doubled, the fitting values are the same. Therefore, we may draw a conclusion that our calculated results are available.

Second, the microindentation test of single crystal Tungsten [3] is simulated. The material parameters are $E=410 \mathrm{GPa}, v=0.278$ [44], $\sigma_{0}=410 \mathrm{MPa}, H_{0}=3160 \mathrm{Mpa}$ [3], $n=0.186$ for (100) and (111), $n=0.245$ for (110) and $c=0$. The value of the hardening exponent $n$ is obtained by fitting the value of $H_{0}$. The results are shown in Figs. 6-8. The numbers in parentheses represent the orientation of the crystallographic plane onto which the indenter is pressed. The numbers in brackets represent the diagonal orientation of the Vickers' indenter. The FEM results based on the plastic strain curl theory simulate the experimental data successfully. The similar linear laws as the Copper samples are shown between the squared hardness $H^{2}$ and the inverse of the contacting depth $1 / h$. It can be seen that the line slopes in

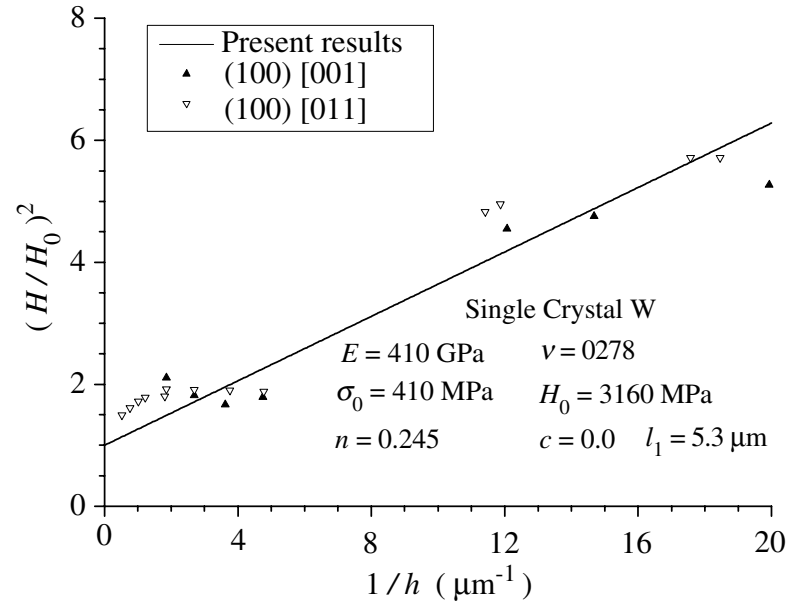

Fig. 7. Simulation of the microindentation test on single crystal Tungsten. (100) plane is indented 


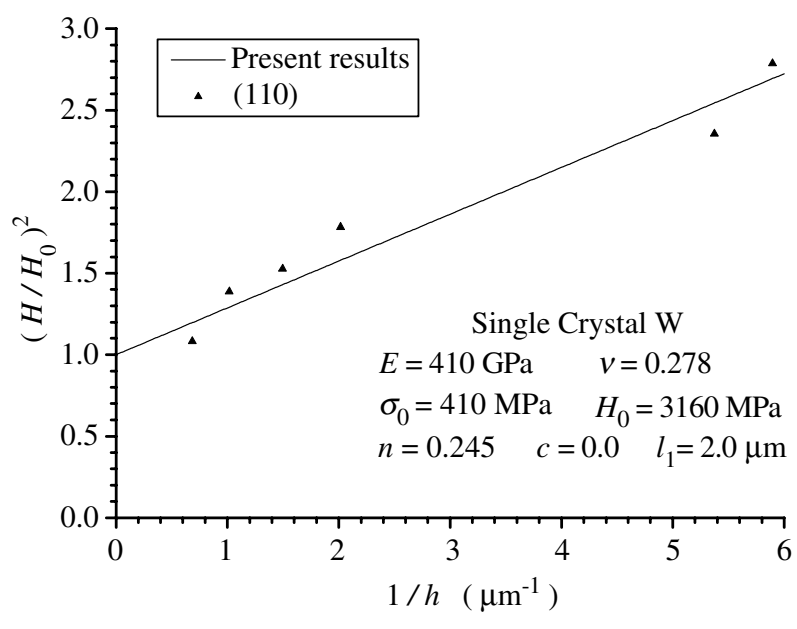

Fig. 8. Simulation of the microindentation test on single crystal Tungsten. (110) plane is indented

Figs. $6-8$ are about $0.269,0.263$ and 0.287 , respectively, i.e. the values are very close to each other. The observation of line slopes seems to imply that the relation between the squared hardness $H^{2}$ and the inverse of contacting depth $1 / h$ has no dependence on the factors such as the grain size, the crystalline orientation, and so on. The fitting values of the length scales are quite different in case of different crystalline orientations. This phenomenon shows the anisotropy of a single crystal. Hence, a more accurate study should be based on a theory of anisotropic plasticity.

\section{Plane-strain crack tip field}

For the FEM formulation of a plane-strain problem, suppose that the $z$-axis is identical with the crack front, the $y$-axis perpendicular to the crack surface and the positive half of the $x$-axis lying ahead of the crack, as shown in Fig. 9.

The non-vanishing displacement and micro rotation components in a plane-strain problem are

$u_{x}=u_{x}(x, y), \quad u_{y}=u_{y}(x, y), \quad \omega_{z}=\omega_{z}(x, y)$.

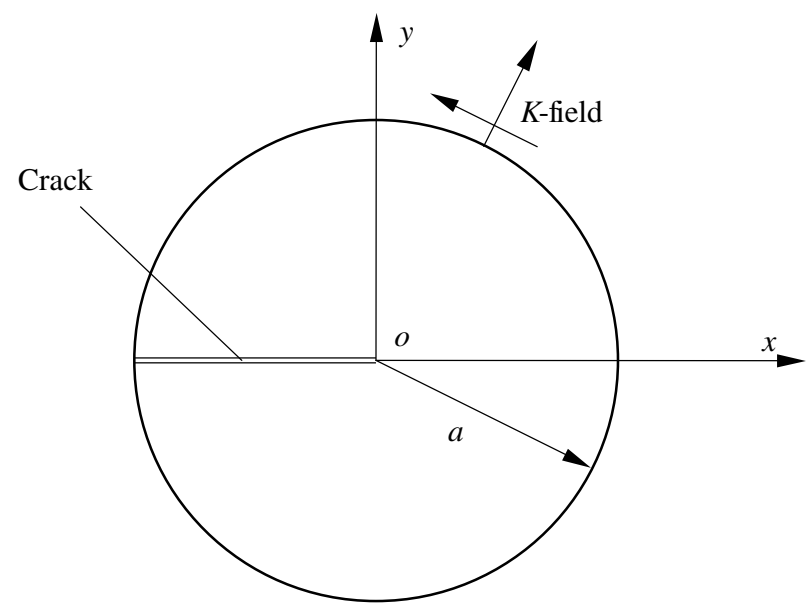

Fig. 9. The calculated domain. The crack tip field is induced by a linear elastic mode-I $k$-field 
The $J$-integral of a plane-strain mode-I crack is

$J_{1}=\int_{\Gamma}\left(w n_{1}-n_{\alpha} \sigma_{\alpha \beta} u_{\beta, 1}\right) \mathrm{d} \Gamma$,

where $\Gamma$ is a contour starting from the lower surface of the crack and ending at the upper one, $n_{\alpha}$ is the normal of $\Gamma, \alpha, \beta=1,2, w$ is the strain energy density and

$w=\int \sigma_{i j} \mathrm{~d} \varepsilon_{i j}+\int m_{i j} \mathrm{~d} \chi_{i j}$.

The calculated domain was a circular domain centered at the crack tip as shown in Fig. 9. The radius of the circular domain was $a$. The classical mode-I $K$-field was imposed on the outer boundary of this domain. The crack surface was supposed to be traction-free for both the stresses and couple stresses. The elastic stress intensity factor, $K_{\mathrm{I}}$, of the remotely applied field increased monotonically.

An eight-noded isoparametric element was chosen and each node had three degrees of freedom. The value of $l_{1} / a$ was about $10^{-7}$ to $10^{-3}$, and the size of the smallest elements was less than $10^{-3} l_{1}$. Efforts were taken to make the ratio of the length to the width of the elements approximately unity. Only the upper half of the domain was calculated due to the symmetry condition. The half domain was divided circumferentially into 12 portions and radially into $N_{r}$ portions, respectively. In order to investigate the influence of the mesh, the value of $N_{r}$ was taken as 50 and 100, respectively. The calculated results are shown in Fig. 10. It is evident that results when $N_{r}=50$ and 100 are almost identical, so $N_{r}$ is taken as 50 in the following calculations.

A plane-strain finite element program was developed. In order to verify the reliability of the present program, the case of conventional plasticity was investigated first. Taking the material length scale $l_{1}$ as zero, the comparison was made with the result of Chen and Wang [34]. Both the two results are plotted in Fig. 11. The parameters are chosen the same as in Chen and Wang [34] i.e., the reference length scale $l_{0}=10^{-3} a$, the ratio of yield strength to Young's modulus $\sigma_{0} / E=0.2 \%$, Poisson's ratio $v=0.3$, hardening exponent $n=0.2$ and stress intensity factor $K_{I}=20 l_{0}^{1 / 2}$. In addition, the dimensionless constant $c$ in Eq. (2.18.3) is taken as zero. The two results are almost identical.

Then the calculation was performed in the case of $l_{1} / a=10^{-3}, \sigma_{0} / E=0.2 \%, v=0.3$, $n=0.2, K_{I}=10 \sigma_{0} l_{1}^{1 / 2}$ and $c=0$.

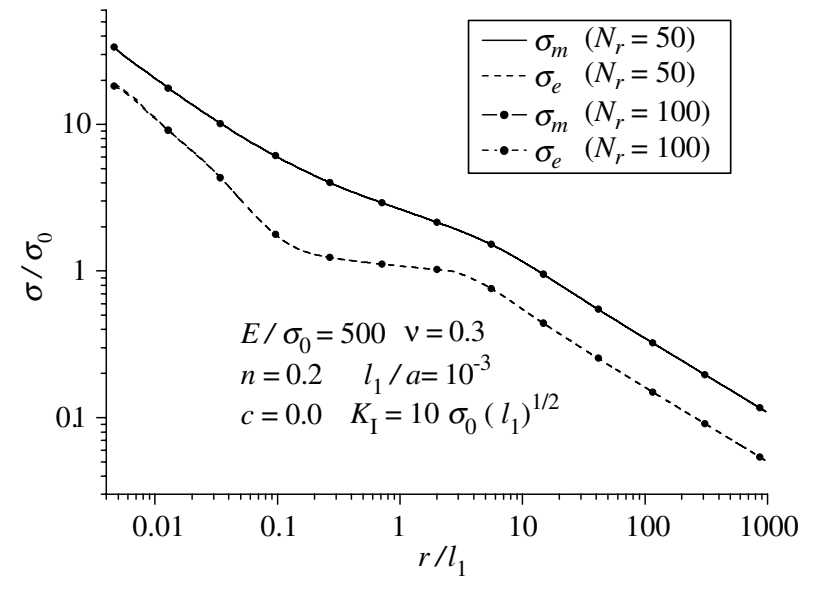

Fig. 10. Calculated equivalent stress $\sigma_{e}$ and mean stress $\sigma_{m}$ with different mesh density 


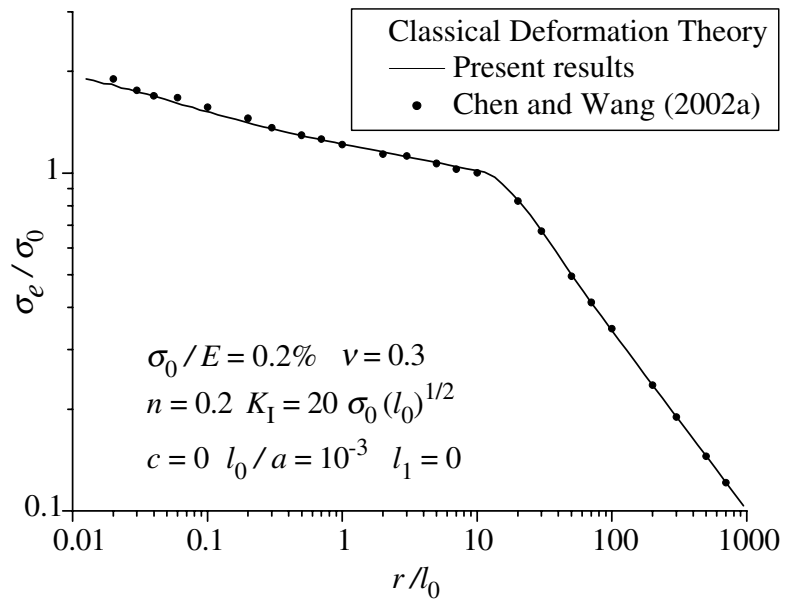

Fig. 11. Comparison with the result by Chen and Wang [31]. The calculation was carried out with the conventional theory of plasticity

The calculated stresses ahead of the crack tip (at polar angle $\theta=3.18^{\circ}$ ) are plotted in Figs. 12 and 13. Figure 12 shows the distribution of normalized mean stress $\sigma_{m} / \sigma_{0}$ and equivalent stress $\sigma_{e} / \sigma_{0}$ versus normalized distance $r / l_{1}$ from the crack tip. Figure 13 shows the distribution of normalized stress components $\sigma_{r r} / \sigma_{0}$ and $\sigma_{\theta \theta} / \sigma_{0}$ versus $r / l_{1}$. It can be seen that the size of the plastic zone (the range in which $\sigma_{e} / \sigma_{0} \geq 1$ ) is a bit larger than $5 l_{1}$. The elastic K-field exists beyond the plastic zone. The curves are all straight lines with a slope of $-1 / 2$ in the elastic zone. As the distance from the crack tip $r$ decreases, the curves are transited into the more even straight lines. This zone, ranging from about $0.2 l_{1}$ to $5 l_{1}$, is the range of HRR type field. The calculated results of plastic strain curl theory and conventional theory are identical in both elastic zone and HRR zone, which means that the effect of geometrically necessary dislocation density (or, in other words, plastic strain curl) is negligible in these two ranges. When $r<0.2 l_{1}$, the density of geometrically necessary dislocations (plastic strain curl) plays an evident role. Therefore, the calculated results with plastic strain curl effect are much higher than the results with conventional plasticity in that range. This means that the calculated stress level at the crack tip will be elevated greatly by the effect of plastic strain curl. By the way, the slope of the mean stress curve is about $-1 / 2$ near the crack tip while the equivalent stress curve is more inclined. This implies that the singularity of mean stress is the square root singularity and the singularity of equivalent stress is higher than the square root singularity. However, since the

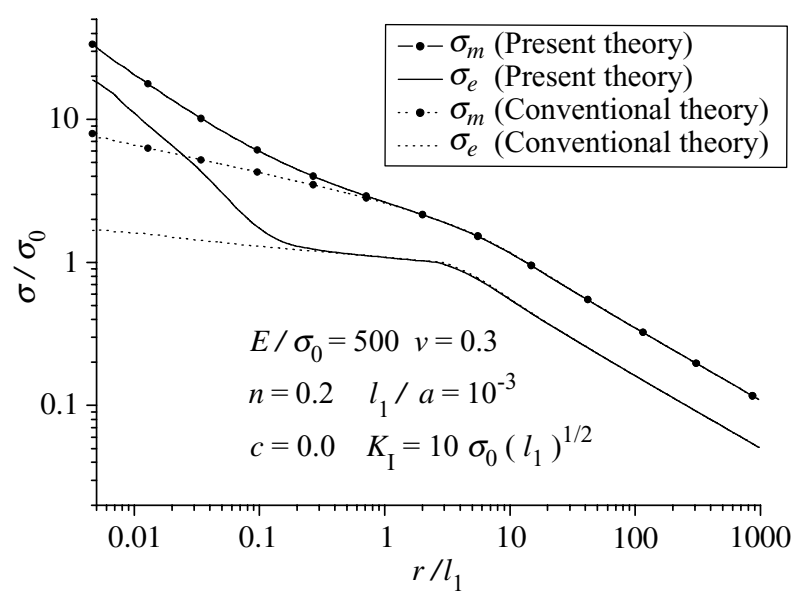

Fig. 12. Normalized mean stress $\sigma_{m} / \sigma_{0}$ and effective stress $\sigma_{e} / \sigma_{0}$ versus normalized distance $r / l_{1}$ from the crack tip. The results are presented for both plastic strain curl theory and conventional theory 


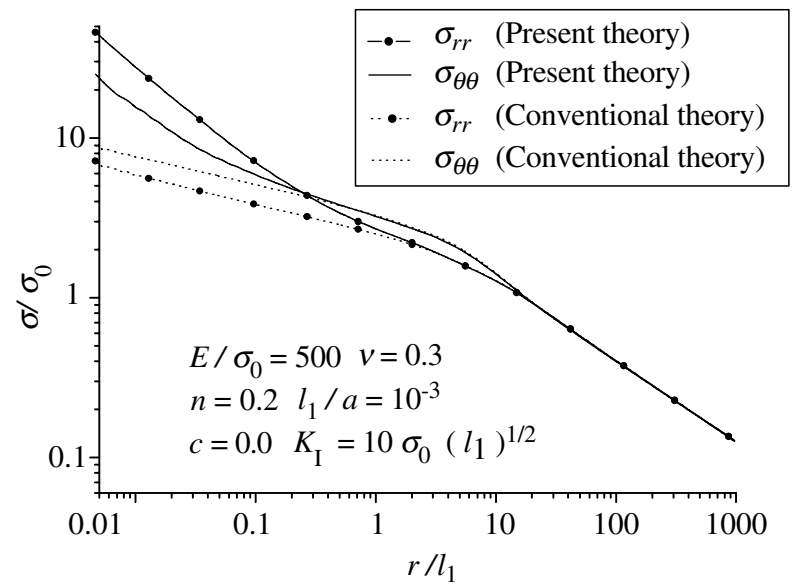

Fig. 13. Normalized stress components $\sigma_{r r} \sigma_{0}$ and $\sigma_{\theta \theta} / \sigma_{0}$ versus normalized distance $r / l_{1}$ from the crack tip. The results are presented for both plastic strain curl theory and conventional theory

mean stress is at least one time greater than the equivalent stress in the calculated range, the slopes of stress component curves are still nearly $-1 / 2$.

Figure 14 is the plot of the normalized J-integral $J E /\left[\left(1-v^{2}\right) K_{I}^{2}\right]$ versus normalized distance $r / l_{1}$ from the crack tip. The calculated contours are 100 circles centered at the crack tip with different radii. It can be observed that the J-integral is almost path independent in the whole range. This result is clearly different from the result by Xia et al. [35]. In that work, the $J$-integral is path independent only in the elastic and HRR type zones. The difference may be caused by the fact that the deformation was not decomposed into elastic and plastic parts in the simplified theory proposed by Xia et al. [35].

The influence of the exponent $n$ is shown in Fig. 15. The value of $n$ has been taken to be 0.1 , 0.2 and 0.3 , respectively. The other parameters are chosen as $l_{1} / a=10^{-3}, \sigma_{0} / E=0.2 \%$, $v=0.3, K_{I}=10 \sigma_{0} l_{1}^{1 / 2}$, and $c=0$. It can be seen that the greater the value of the hardening exponent $n$ is, the higher the stress level in the plastic zone will be. In the range of HRR type field, the greater value of $n$ leads to the steeper inclination of the curve. In fact, the stress singularity of HRR type field is $r^{-n /(n+1)}$. The slopes of three curves are approximately the same in the range where plastic strain curl plays an evident role. Therefore, the stress singularities are also the same in the three cases.

The distribution of normalized equivalent stress $\sigma_{e} / \sigma_{0}$ versus $r / l_{1}$ under different load levels is plotted in Fig. 16. The value of stress intensity factor $K_{I}$ has been taken as $10 \sigma_{0} l_{1}^{1 / 2}, 20 \sigma_{0} l_{1}^{1 / 2}$

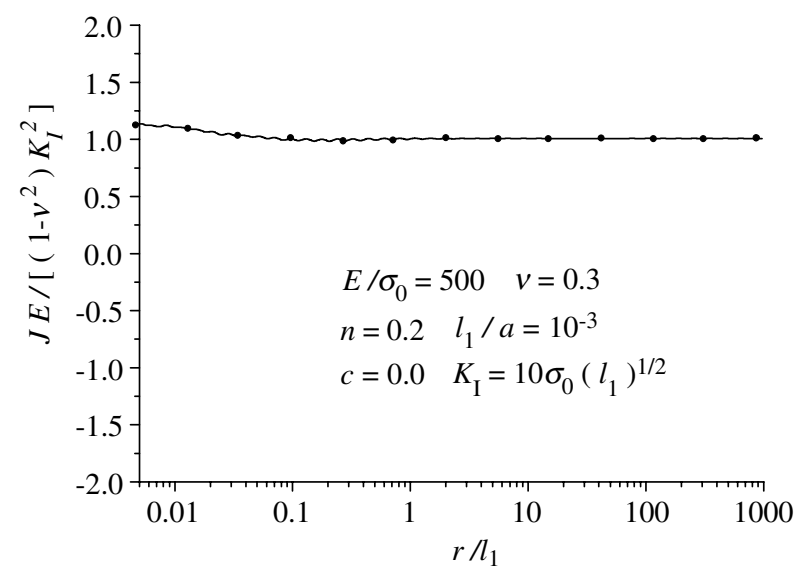

Fig. 14. Normalized J-integral $J E /$ $\left[\left(1-v^{2}\right) K_{1}^{2}\right] \quad$ versus normalized distance $r / l_{1}$ from the crack tip 


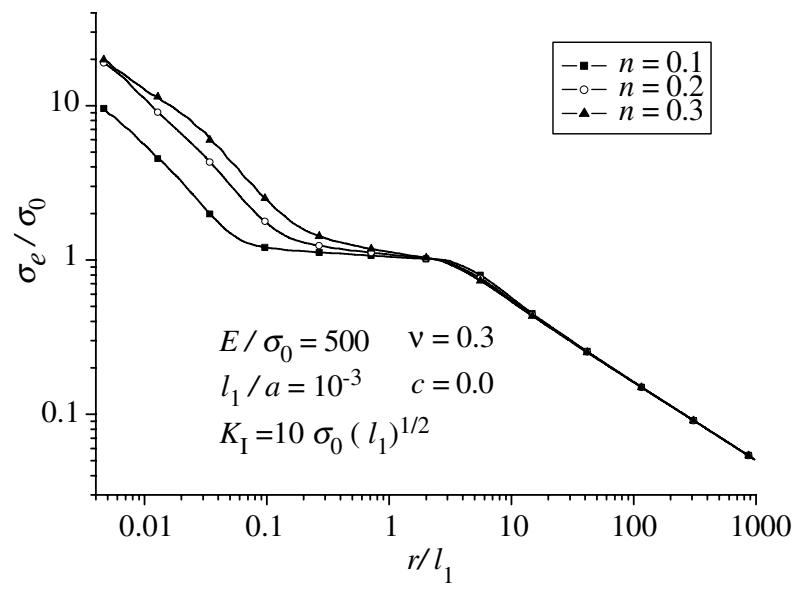

Fig. 15. Normalized equivalent stress $\sigma_{e} / \sigma_{0}$ versus normalized distance $r / l_{1}$ from the crack tip. The results are presented for various values of the hardening exponent $n$

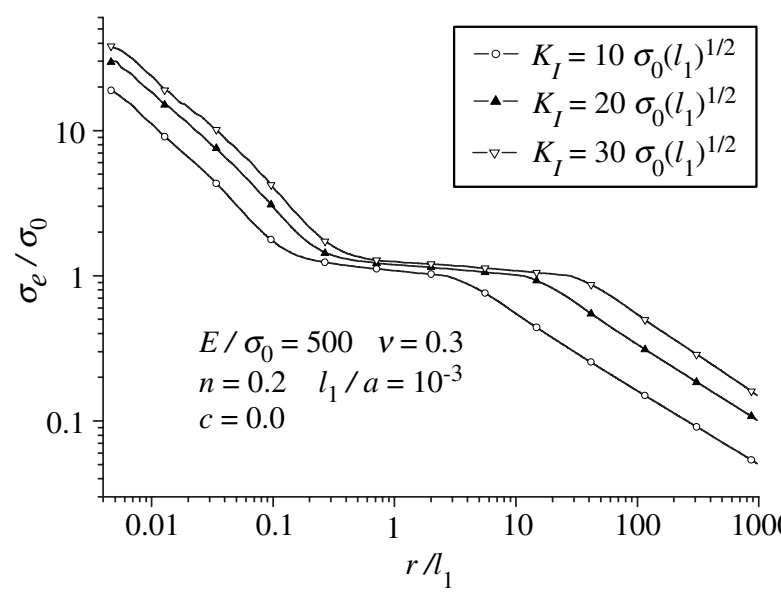

Fig. 16. Normalized mean stress $\sigma_{e} / \sigma_{0}$ versus normalized distance $r / l_{1}$ from the crack tip. The results are presented for various values of the stress intensity factor $K_{1}$

and $30 \sigma_{0} l_{1}^{1 / 2}$, respectively. The other parameters are chosen as $l_{1} / a=10^{-3}, \sigma_{0} / E=0.2 \%$, $v=0.3, n=0.2$ and $c=0$. Apparently, the stress level increases as the external load increases. Accordingly, the sizes of the plastic zone, HRR type zone and plastic-strain-curl-dominating zone increase also. The slopes of the three curves are almost identical in all ranges of the calculated domain. It seems that stress singularity is independent on the load level.

\section{Conclusion}

The plastic strain curl theory has been proposed in this paper in order to consider the effect of dislocation density. According to the viewpoints of material science, work hardening of metals is caused by the accumulation of dislocations and the increase of dislocation density. Plastic strain curl plays the key role in the influence of dislocation density. The measure of plastic strain curl is incorporated as an internal variable to elevate the tangent modulus. In this way, finite element formulations of microindentation tests and the plane-strain mode-I crack tip field are developed. The indentation size effect is successfully simulated. The values of material length scale $l_{1}$ for polycrystal copper, single crystal copper and single crystal tungsten are obtained. The crack tip field for small scale yielding condition has been investigated. The 
numerical results show the elevation of the stress level, which may explain the experimental results of Elssner et al. [7]. It is observed that near the crack tip the singularity of the mean stress seems to be of the square-root singularity while the singularity of equivalent stress is higher than the square root singularity. The J-integral is path independent in the calculated domain. Neither the hardening exponent $n$ nor the stress intensity factor can influence the stress singularity in the range where the plastic strain curl plays an evident role.

\section{Acknowledgements}

The present work is supported by the National Natural Science Foundation of China (No. 10272103).

\section{References}

[1] Ashby, M. F.: The deformation of plastically non-homogeneous alloys. Phil. Mag. 21, 399 (1970).

[2] Nix, W. D., Gao, H.: Indentation size effects in crystalline materials: a law for strain gradient plasticity. J. Mech. Phys. Solids 46, 411-425 (1998).

[3] Stelmashenko, N. A., Walls, M. G., Brown, L. M., Milman, Y. V.: Microindentation on W and Mo oriented single crystals: an STM study. Acta Metall. Mater. 41, 2855-2865 (1993).

[4] De Guzman, M. S., Neubauer, G., Flinn, P., Nix, W. D.: The role of indentation depth on the measured hardness of materials. Mater. Res. Soc. Symp. Proc. 308, 613-618 (1993).

[5] Ma, Q., Clarke, D. R.: Size dependence hardness in silver single crystals. J. Mater. Res. 10, 853-863 (1995).

[6] McElhaney, K. W., Vlassak, J. J., Nix, W. D.: Determination of indenter tip geometry and indentation contact area for depth-sensing indentation experiments. J. Mater. Res. 13, 1300-1306 (1998).

[7] Elssner, G., Korn, D., Ruehle, M.: The influence of interface impurities on fracture energy of UHV diffusion bonded metal-ceramic bicrystals. Scripta Metall. Mater. 31, 1037 (1994).

[8] Hutchinson, J. W.: Linking scales in mechanics. In: Advances in fracture research (Karihaloo, B. L., Mai, Y. W., Ripley, M. I., Ritchie, R. O., eds.), pp. 1. New York: Pergamon Press 1997.

[9] Fleck, N. A., Hutchinson, J. W.: A phenomenological theory for strain gradient effects in plasticity. J. Mech. Phys. Solids 41, 1825 (1993).

[10] Fleck, N. A., Muller, G. M., Ashby, M. F., Hutchinson, J. W.: Strain gradient plasticity: theory and experiment. Acta Metall. Mater. 42, 475 (1994).

[11] Shu, J. Y., Fleck, N. A.: The prediction of a size effect in microindentation. Int. J. Solids Struct. 35, 1363 (1998).

[12] Huang, Y., Zhang, L., Guo, T. F., Hwang, K. C.: Near-tip fields for cracks in materials with strain-gradient effects. In: Proc. IUTAM Symp. on Nonlinear Analysis of Fracture (Willis, J. R., ed.), pp. 231. Cambridge: Kluwer 1995.

[13] Huang, Y., Zhang, L., Guo, T. F., Hwang, K. C.: Mixed mode near-tip fields for cracks in materials with strain-gradient effects. J. Mech. Phys. Solids 45, 439-465 (1997).

[14] Xia, Z. C., Hutchinson, J. W.: Crack tip fields in strain gradient plasticity. J. Mech. Phys. Solids 44, 1621-1648 (1996).

[15] Chen, J. Y., Huang, Y., Hwang, K. C.: Mode-I and mode-II plane-stress near-tip fields for cracks in materials with strain gradient effects. Key Engng Mater. 145, 19-28 (1998).

[16] Huang, Y., Zhang, L., Guo, T. F., Hwang, K. C.: Fracture of materials with strain gradient effects. In: Advances in fracture research (Karihaloo, B. L., Mai, Y. W., Ripley, M. I., Ritchie, R. O., eds.), pp. 2275-2286. Amsterdam: Pergamon Press 1997.

[17] Fleck, N. A., Hutchinson, J. W.: Strain gradient plasticity. In: Advances in applied mechanics (Hutchinson, J. W., Wu, T. Y., eds.), vol. 33, pp. 295. New York: Academic Press 1997.

[18] Begley, M. R., Hutchinson, J. W.: The mechanics of size-dependent indentation. J. Mech. Phys. Solids 46, 2049-2068 (1998). 
[19] Wei, Y., Hutchinson, J. W.: Steady-state crack growth and work of fracture for solids characterized by strain gradient plasticity. J. Mech. Phys. Solids 45, 1253-1273 (1997).

[20] Chen, J. Y., Wei, Y., Huang, Y., Hutchinson, J. W., Hwang, K. C.: The crack tip fields in strain gradient plasticity: the asymptotic and numerical analyses. Engng Fract. Mech. 64, 625-648 (1999).

[21] Shi, M. X., Huang, Y., Hwang, K. C.: Fracture in the higher-order elastic continuum. J. Mech. Phys. Solids 48, 2513-2538 (2000).

[22] Gao, H., Huang, Y., Nix, W. D.: Modeling plasticity at the micrometer scale. Naturwissenschaften 86, 507-515 (1999).

[23] Gao, H., Huang, Y., Nix, W. D., Hutchinson, J. W.: Mechanism-based strain gradient plasticityI. Theory. J. Mech. Phys. Solids 47, 1239 (1999).

[24] Huang, Y., Gao, H., Nix, W. D., Hutchinson, J. W.: Mechanism-based strain gradient plasticityII. Analysis. J. Mech. Phys. Solids 48, 99 (2000).

[25] Huang, Y., Xue, Z., Gao, H., Nix, W. D., Xia, Z. C.: A study of micro-indentation hardness tests by mechanism-based strain gradient plasticity. J. Mater. Res. 15, 1786 (2000).

[26] Shi, M. X., Huang, Y., Gao, H., Hwang, K. C.: Non-existence of separable crack tip field in mechanism-based strain gradient plasticity. Int. J. Solids Struct. 37, 5995-6010 (2000).

[27] Jiang, H., Huang, Y., Zhuang, Z., Hwang, K. C.: Fracture in mechanism-based strain gradient plasticity. J. Mech. Phys. Solids 49, 979-993 (2001).

[28] Acharya, A., Bassani, J. L.: In: Micromechanics of plasticity and damage of multiphase materials, pp. 1-8, IUTAM Symp., Paris, August 29-September 1, 1995.

[29] Chen, S. H., Wang, T. C.: A new hardening law for strain gradient plasticity. Acta Mater. 48, 3997-4005 (2000).

[30] Chen, S. H., Wang, T. C.: Strain gradient theory with couple-stress for crystalline solids. Eur. J. Mech. A / Solids 20, 739-756 (2001).

[31] Chen, S. H., Wang, T. C.: A new deformation theory for strain gradient effects. Int. J. Plasticity 18, 971-995 (2002).

[32] Chen, S. H., Wang, T. C.: Mode-I crack tip field with strain gradient effects. Acta Mech. Solida Sinica 13, 290-298 (2000).

[33] Chen, S. H., Wang, T. C.: Mode-I and mode-II crack tip asymptotic fields with strain gradient effects. Acta Mech. Sinica 17, 269-280 (2001).

[34] Chen, S. H., Wang, T. C.: Finite element solutions for plane-strain mode-I crack with strain gradient effects. Int. J. Solids Struct. 39, 1241-1257 (2002).

[35] Xia, S., Wang, T. C., Chen, S. H.: Crack tip field and J-integral with strain gradient. Acta Mech. Sinica (in press).

[36] Kröner, E.: Continuum theory of defects. In: Physics of defects (Balian, R. et al., eds.), pp. 219316. New York: North-Holland 1981.

[37] Cosserat, E., Cosserat, F.: Theorie des corps deformables. Paris: Herman et fils 1909.

[38] Toupin, R.: Elastic materials with couple-stresses. Arch. Rat. Mech. Anal. 11, 385 (1962).

[39] Mindlin, R. D.: Influence of couple-stress on stress concentrations. Exp. Mech. 3, 1 (1963).

[40] Mindlin, R. D.: Microstructure in linear elasticity. Arch. Rat. Mech. Anal. 16, 51-78 (1964).

[41] Schaefer, H.: Das Cosserat-Kontinuum. ZAMM 47, 485 (1967).

[42] Eringen, A. C.: Theory of micropolar elasticity. In: Fracture, an advanced treatise (Leibowitz, H., ed.), pp. 621. New York: Academic Press 1968.

[43] McLean, D.: Mechanical properties of metals. New York: Wiley 1962.

[44] Hirth, J. P., Lothe, J.: Theory of dislocations, 2nd ed. New York: Wiley 1982.

Authors' address: S. Xia and T. Wang, LNM, Institute of Mechanics, Chinese Academy of Sciences, Beijing 100080, P.R. China (E-mail: tcwang@imech.ac.cn) 